\title{
Internetsucht
}

\section{Wie viele Jugendliche sind betroffen?}

\author{
Neben stoffgebundenen Süchten rückt der pathologische Internetgebrauch in den Fokus der Forschung. \\ Wie viele Jugendliche sind davon betroffen und welche psychopathologischen und psychosozialen Faktoren \\ hängen damit zusammen?
}

U nzählige Stunden am Computer, immer schlechtere Schulleistungen, keine Zeit für Freunde, Missstimmung und Reizbarkeit, wenn man nicht online ist: Pathologischer Internetgebrauch bei Jugendlichen ist ein Thema, dem immer mehr Forscher Beachtung schenken. Mit zunehmender Verfügbarkeit des Mediums in deutschen Kinderzimmern nahm auch die Publikationsrate in den vergangenen Jahren zu. Dennoch bleibt weiterhin unklar, in welcher Größenordnung dieses Problem bei Jugendlichen auftritt. Angaben zu Prävalenzraten in Europa schwanken zwischen $2 \%$ und $18 \%$ [1]. Häufig sind die untersuchten Stichproben nicht repräsentativ und die eingesetzten Erhebungsverfahren nicht ausreichend validiert. Dazu kommt, dass es derzeit keine einheitliche Definition für Internetabhängigkeit gibt [2].

Forscher der Universität Heidelberg analysierten nun dazu Daten aus einer randomisiert-kontrollierten Multicen- terstudie, die in elf europäischen Staaten durchgeführt wurde [3]. Europaweit nahmen an der Studie über 12.000 Schüler im Alter zwischen 14 und 16 Jahren teil.

\section{Ab wann ist es pathologisch?}

Die deutsche Stichprobe erfasste 1.435 Schüler aus dem Rhein-Neckar-Kreis, die mittels verschiedener Fragebögen untersucht wurden, u.a. dem Young Diagnostic Questionnaire. Dieser orientiert sich an den DSM-IV-Kriterien für pathologisches Glücksspiel und wird häufig als Screening für den pathologischen Internetgebrauch benutzt (Tab. 1). Die befragten Schüler wurden in drei Gruppen eingeteilt: Wer weniger als zwei Fragen mit „Ja“ beantwortete galt als unauffälliger User, bei drei bis vier erfüllten Kriterien sahen die Wissenschaftler die Internetnutzung als problematisch, bei fünf und mehr Kriterien als pathologisch an.

\section{Symptome des pathologischen Internetgebrauchs [5]}

— Beschäftigen Sie sich nahezu ausschließlich mit dem Internet (über vergangene Onlineaktivitäten nachdenken oder sich die nächste Onlinesitzung im voraus vorstellen)?

- Empfinden Sie das Bedürfniss, das Internet immer länger zu nutzen, um damit zufrieden sein zu können?

- Haben Sie mehrfach erfolglos versucht, Ihre Zeit im Internet zu kontrollieren oder zu reduzieren oder den Internetgebrauch zu beenden?

_ Fühlen Sie sich ruhelos, launisch, deprimiert und reizbar, wenn Sie Ihren Internetgebrauch zu reduzieren oder zu beenden versuchen?

_ Bleiben Sie länger online als zunächst beabsichtigt?

_ Haben Sie wegen des Internets bereits den Verlust bedeutsamer Beziehungen oder der Arbeitsstelle oder von Bildungs- bzw. Karrierechancen aufs Spiel gesetzt?

_ Haben Sie Familienmitglieder, Therapeuten oder andere über die Intensität Ihres Internetgebrauchs belogen?

_ Nutzen Sie das Internet als Möglichkeit, Problemen zu entkommen oder der Erleichterung schlechter Stimmungen (z. B. Gefühle von Hilflosigkeit, Schuld, Angst und Niedergeschlagenheit)?

Nach: Young KS. CyberPsychology and Behavior 1998; 1(3):237-244

\section{Mit Depression, Selbstverletzung und Suizidalität assoziiert}

Die Prävalenz für pathologischen Internetgebrauch lag in dieser Untersuchung bei $4,8 \%$. Eine problematische Nutzung fanden die Forscher bei $14,5 \%$ und $80,7 \%$ zeigten einen unauffälligen Internetkonsum.

Auffällige Jugendliche hatten im Vergleich zu unauffälligen signifikant höhere Depressionsraten und wiesen häufiger selbstverletzendes und suizidales Verhalten auf. Diese Erkenntnisse müssten dazu führen nicht erst bei pathologischem, sondern bereits bei problematischem Konsum präventiv einzugreifen, um psychische Probleme rechtzeitig zu erkennen, so das Fazit der Heidelberger Forscher.

Als Risikofaktoren für eine Internetsucht zählten laut europaweiter Auswertung unter anderem eine städtische Umgebung und nahezu alle Variablen, die in Zusammenhang mit der Eltern-KindBeziehung stehen. Als besonders gefährdet identifizierten die Wissenschaftler Schüler, die nicht bei ihrer Familie lebten, unter einer geringen elterlichen Fürsorge standen oder arbeitslose Eltern hatten [4].

Dr. Laura Vöhringer

1. Johansson et al. Scand J Psychol 2004; 45(3): 223-9

2. Petersen et al. Endbericht an das Bundesministerium für Gesundheit 2010, Deutsches Zentrum für Suchtfragen des Kindes- und Jugendalters, Hamburg

3. Fischer $\mathrm{G}$ et al. Prax Kinderpsychol Kinderpsychiatr 2012; 61(1):16-31

4. Durkee T et al. Addiction 2012; 107(12):221022

5. Petersen KU et al. Fortschr Neurol Psychiat 2009; 77:263-71

Vortrag "Prävalenz und psychosoziale Korrelate pathologischer Internetnutzung in Europa", DGKJP-Kongress, 6.3.2013, Rostock 